\title{
Video Article \\ High Resolution Quantitative Synaptic Proteome Profiling of Mouse Brain Regions After Auditory Discrimination Learning
}

\author{
Angela Kolodziej ${ }^{1}$, Karl-Heinz Smalla ${ }^{1}$, Sandra Richter ${ }^{2}$, Alexander Engler ${ }^{1}$, Rainer Pielot ${ }^{1,3}$, Daniela C. Dieterich ${ }^{3}$, Wolfgang Tischmeyer $^{1}$, \\ Michael Naumann ${ }^{2}$, Thilo Kähne ${ }^{2}$ \\ ${ }^{1}$ Leibniz Institute for Neurobiology (LIN) \\ ${ }^{2}$ Institute of Experimental Internal Medicine, Medical School, Otto von Guericke University Magdeburg \\ ${ }^{3}$ Institute of Pharmacology and Toxicology, Medical School, Otto von Guericke University
}

Correspondence to: Thilo Kähne at kaehne@med.ovgu.de

URL: https://www.jove.com/video/54992

DOI: doi: $10.3791 / 54992$

Keywords: Neuroscience, Issue 118, neurobiology, auditory learning, synaptosomes, quantitative mass spectrometry, label-free quantification, phospho-proteomics, bioinformatics, meta-analysis

Date Published: $12 / 15 / 2016$

Citation: Kolodziej, A., Smalla, K.H., Richter, S., Engler, A., Pielot, R., Dieterich, D.C., Tischmeyer, W., Naumann, M., Kähne, T. High Resolution Quantitative Synaptic Proteome Profiling of Mouse Brain Regions After Auditory Discrimination Learning. J. Vis. Exp. (118), e54992, doi:10.3791/54992 (2016).

\section{Abstract}

The molecular synaptic mechanisms underlying auditory learning and memory remain largely unknown. Here, the workflow of a proteomic study on auditory discrimination learning in mice is described. In this learning paradigm, mice are trained in a shuttle box Go/NoGo-task to discriminate between rising and falling frequency-modulated tones in order to avoid a mild electric foot-shock. The protocol involves the enrichment of synaptosomes from four brain areas, namely the auditory cortex, frontal cortex, hippocampus, and striatum, at different stages of training. Synaptic protein expression patterns obtained from trained mice are compared to naïve controls using a proteomic approach. To achieve sufficient analytical depth, samples are fractionated in three different ways prior to mass spectrometry, namely 1D SDS-PAGE/in-gel digestion, insolution digestion and phospho-peptide enrichment.

High-resolution proteomic analysis on a mass spectrometer and label-free quantification are used to examine synaptic protein profiles in phospho-peptide-depleted and phospho-peptide-enriched fractions of synaptosomal protein samples. A commercial software package is utilized to reveal proteins and phospho-peptides with significantly regulated relative synaptic abundance levels (trained/naïve controls). Common and differential regulation modes for the synaptic proteome in the investigated brain regions of mice after training were observed. Subsequently, meta-analyses utilizing several databases are employed to identify underlying cellular functions and biological pathways.

\section{Video Link}

The video component of this article can be found at https://www.jove.com/video/54992/

\section{Introduction}

Learning is based on the formation of memory traces and their maintenance. It is widely accepted that one underlying mechanism may represent an activity-dependent formation of new and/or rearrangement of existing synaptic contacts between neurons. On the molecular level, various protein modifications, subcellular relocalizations and changes in the turnover of synaptic proteins have been described ${ }^{1-4}$ (Lamprecht, 2004 \#8). However, most studies so far focused on selected proteins rather than on the global but complex synaptic proteome composition. The present approach allows an unbiased screening for synaptic proteome changes in mouse brain regions after a learning experiment. It is suitable to render time-point dependent molecular snapshots of the learning-induced reorganization of the synaptic architecture. The described workflow requires a particular teamwork of different specialists in animal behavior, protein biochemistry, mass spectrometry and bioinformatics.

The chosen learning paradigm, i.e. frequency-modulated tone discrimination (FMTD), is a well-characterized auditory discrimination task in rodents ${ }^{5}$. Learning and long-term memory formation in this shuttle box Go/No-Go-task involves mechanisms depending on increased cortical dopamine signaling and protein synthesis. Accordingly, recent proteomic studies on gerbils and mice revealed dopamine- and learning-induced plastic rearrangements of synaptic components in cortical, but also in more basal brain regions that supposedly interact during FMTD learning and memory ${ }^{6-8}$. This illustrates that memory formation involves a complex interplay of various brain regions and thus, might be differentially regulated within these regions on the proteome level. Therefore, dissection of selected cortical and subcortical mouse brain regions is included in the workflow.

Furthermore, the reliable characterization even of weak changes in synaptic protein composition requires an enrichment of pre- and postsynaptic compartments rather than the analysis of homogenates or crude membrane fractions ${ }^{9}$. Therefore, the preparation of synaptosomes utilizing established protocols prior to proteomic analysis is described in order to increase the detection level and the dynamic range for synapse-specific proteins $^{10,11}$. 
An essential prerequisite to use label-free high-resolution mass spectrometry for quantitative purposes is a high degree of similarity of protein samples. As rather minor changes in synaptic protein composition are expected to occur after learning, a label-free approach will be appropriate to compare corresponding protein samples obtained from trained and naïve mice. Alternatively, condition-specific label strategies of proteins/ peptides using stable isotopes (e.g. TMT, iTRAQ, ICPL and SILAC) as well as MS2-based label free quantification (SWATH) are useful, but they are more expensive than the chosen label-free approach or need special mass spectrometric hardware.

Since proteomic screenings often yield complex data sets, bioinformatic processing is recommended for appropriate data interpretation. Additional meta-analyses may support a better understanding of potential molecular mechanisms underlying paradigm-related changes and the identification of involved key cellular processes and signaling pathways. Appropriate methodologies are also described below.

\section{Protocol}

All procedures including animal subjects were performed in accordance with the regulations of the German Federal Law, the respective EU regulations and $\mathrm{NIH}$ guidelines, and have been approved by the ethics committee of the Landesverwaltungsamt Sachsen/Anhalt (42502-2-1102 IfN).

\section{Auditory Learning}

1. Auditory discrimination learning in the shuttle box (FMTD paradigm) Note: Always wear gloves while handling the mice.

1. House C57BI6/J mice in groups of three or four with free access to food pellets and tap water in clear polycarbonate cages. Maintain a $12 \mathrm{hr}$ light:dark cycle in the animal facility. If animals are received from another lab or from a company allow at least one week of acclimation and settling in.

2. Perform one shuttle box training session per day.

1. Take the mouse from its home cage in the animal facility and place it in a dimly lit shuttle box within a sound proof chamber.

2. Use a fully computer-controlled learning schedule for auditory discrimination learning. Begin with a habituation period of 3 min of silence, and then start the training session.

1. Use sequences of the rising tone $(4-8 \mathrm{kHz}, \mathrm{CS}+)$ as the Go-stimulus during Go-trials: The animal has to cross the hurdle within $6 \mathrm{sec}$ of tone presentation (correct response, hit). Punish a miss by a mild foot-shock of $50-300 \mu \mathrm{A}$, delivered via the grid floor of the shuttle box.

2. Use sequences of the falling tone ( $8-4 \mathrm{kHz}, \mathrm{CS}-)$ as the No-Go-stimulus during No-Go-trials: The animal has to remain in the current compartment of the shuttle box during the $6 \mathrm{sec}$ of tone presentation. Punish a false alarm by a mild foot-shock of $50-300 \mu \mathrm{A}$, delivered via the grid floor of the shuttle box.

3. Use intertrial intervals of $205 \mathrm{sec}$.

4. Perform 30 Go-trials and 30 No-Go-trials per session in a pseudo-randomized order, so that one session consists of 60 trials and lasts about $25 \mathrm{~min}$.

3. Put the trained animal back into its home cage in the animal facility.

\section{Brain dissection}

1. Euthanize the animal at the desired time point after a desired number of training sessions using cervical dislocation (e.g. $24 \mathrm{hr}$ after completion of the first session). Decapitate the animal.

2. Quickly dissect the brain via the following steps: Cut first the skin and then the skull with straight scissors along the Sutura sagittalis Completely remove the parts of the bone which cover the brain tissue using strong forceps. Take out the brain with a spattle.

3. For dissection, place brain onto a Petri dish filled with ice. Dissect the auditory cortex, the frontal cortex, the striatum and the hippocampus under a stereomicroscope using a scalpel and a needle.

1. Localize the auditory cortex using visual landmarks on the brain surface such as blood vessels and the shape of the surface (Bregma -2.06 to -3.4 , size rostrocaudal $2 \mathrm{~mm}$, dorsoventral $1.3 \mathrm{~mm}$ ) and bilaterally dissect as a rectangular tissue block with the thickness of the cortex.

2. Dissect the frontal cortex as a brain slice between Bregma 3.56 and 1.54 using the chiasma opticum as a landmark and excluding tissue from bulbus olfactorius.

3. Dissect the striatum as a brain slice between Bregma 1.54 and 0.5 and carefully remove cortical tissue.

4. Dissect the hippocampus by fixing the brain with the needle through the cerebellum and uncoiling the cortex starting at the occipital lobe.

4. Shock-freeze dissected brain samples in liquid nitrogen and store at $-80{ }^{\circ} \mathrm{C}$.

\section{Preparation of Synaptosomes or Alternatively a Postsynaptic-density (PSD)-enriched Fraction}

NOTE: During all procedures, keep samples and buffers at $0-4^{\circ} \mathrm{C}$. Buffers contain freshly diluted protease inhibitor cocktails in order to prevent proteolytic degradation of proteins. If protein phosphorylation is also studied, phosphatase inhibitor cocktails have to be added. All g-values indicated are given as $\mathrm{g}$ (average) throughout the whole protocol.

1. Preparation of a crude membrane fraction (Figure $3 A$ )

1. Transfer dissected brain tissue into a homogenization vessel containing $1 \mathrm{ml}$ ice cold buffer A (5 mM HEPES, $320 \mathrm{mM}$ sucrose, pH 7.4) and homogenize tissue at $900 \mathrm{rpm}$ with 12 strokes. 
2. Centrifuge samples at $1,000 \times \mathrm{g}$ for $10 \mathrm{~min}$. Keep the supernatants.

3. Re-homogenize pellets at the same conditions in the same volume of homogenization buffer as before and centrifuge samples again at $1,000 \times \mathrm{g}$ for $10 \mathrm{~min}$. Combine corresponding supernatants. Discard the pellets $\mathrm{P} 1$, which mainly contain nuclei and cell debris.

4. Spin the combined supernatants for $20 \mathrm{~min}$ at $12,000 \mathrm{x}$. Discard supernatants or use for further fractionation ${ }^{11}$.

5. Resuspend pellets in the same volume of homogenization buffer as before using the homogenizer with 6 strokes at $900 \mathrm{rpm}$ and spin at $12,000 \times \mathrm{g}$ for $20 \mathrm{~min}$. Discard supernatants. The pellets P2 represent the crude membrane fractions.

2. Purification of synaptosomes from crude brain membrane fractions (Figure 3A)

NOTE: Crude brain membrane fractions can be separated into myelin, light membranes, synaptosomes and mitochondria using sucrose density step gradient ultracentrifugation. For this $5 \mathrm{mM}$ Tris/ $\mathrm{HCl} \mathrm{pH} 8.1$ buffers containing sucrose at either $0.32 \mathrm{M}, 1.0 \mathrm{M}$ or $1.2 \mathrm{M}$ concentration are required.

1. While performing the centrifugation to produce the $\mathrm{P} 2$ fractions, prepare sucrose step gradients in the ultracentrifuge tubes. Start with $2.5 \mathrm{ml} 1.0 \mathrm{M}$ sucrose buffer and sublayer with $1.5 \mathrm{ml} 1.2 \mathrm{M}$ sucrose buffer using a glass Pasteur pipet.

2. Re-homogenize $\mathrm{P} 2$ fractions in $0.5 \mathrm{ml}$ of $0.32 \mathrm{M}$ sucrose buffer manually with 6 strokes and load on top of the gradient.

3. Spin at $85,000 \times \mathrm{g}$ for $2 \mathrm{hr}$ in an ultracentrifuge using a swinging bucket rotor.

4. Discard the top $0.32 \mathrm{M}$ sucrose layer including the material at the interface to the $1.0 \mathrm{M}$ sucrose buffer (myelin, light membranes). Collect synaptosomes at the 1.0/1.2 M sucrose buffer interface. The pellet at the bottom of the tube contains mitochondria.

5. Add $0.32 \mathrm{M}$ sucrose buffer to the synaptosomal fraction at 1:1 ratio, mix carefully and spin at $150,000 \times \mathrm{g}$ for $1 \mathrm{hr}$. Synaptosomes are in the pellet and can now be resuspended in a buffer required for further processing.

\section{Preparation of a PSD-enriched fraction (Figure 3B)}

1. Homogenize each specific brain area from a single animal in $100 \mu \mathrm{l}$ extraction buffer $(5 \mathrm{mM} \mathrm{Tris} / \mathrm{HCl} \mathrm{pH} 8.1,0.5 \%$ Triton X-100) in a $200 \mu$ ultracentrifuge tube with a PTFE (polytetrafluorethylene) pestle at 2,000 rpm with 12 strokes.

2. Add $100 \mu \mathrm{L}$ extraction buffer, mix and incubate for $1 \mathrm{hr}$ at $4{ }^{\circ} \mathrm{C}$. Spin down at $100,000 \mathrm{xg}$ for $1 \mathrm{hr}$ and collect the supernatant $\mathrm{S} 1$ carefully with a $200 \mu$ l pipet.

3. Re-homogenize pellet P1 in the same tube with $100 \mu$ l extraction buffer again with a PTFE pestle at 2,000 rpm with 12 strokes

4. Add $100 \mu \mathrm{l}$ extraction buffer and mix well with a pipette and spin at $100,000 \times \mathrm{g}$ for $1 \mathrm{hr}$.

5. Combine the supernatant S2 with S1 to the soluble protein fraction. This fraction contains cytosolic proteins, $0.5 \%$ Triton X-100 soluble membrane proteins and extracellular matrix molecules.

6. Resuspend the remaining pellet in $50 \mu \mathrm{l} 5 \mathrm{mM}$ Tris/ $\mathrm{HCl} \mathrm{pH} \mathrm{8.1.} \mathrm{This} \mathrm{fraction} \mathrm{contains} \mathrm{PSDs,} \mathrm{detergent-resistant} \mathrm{membranes,} \mathrm{insoluble}$ cytoskeletal elements, mitochondria and cell debris including nuclei. It is enriched in PSDs which form the core of postsynaptic structures but also important parts of the presynaptic cytomatrix at the active zone. The factor for enrichment of PSDs is around 4 and the enrichment of PSD components has been demonstrated previously. ${ }^{12}$

\section{Sample Preparation for Mass Spectrometry}

\section{Lysis and sample normalization}

NOTE: Sample normalization concerning the protein concentration is a very crucial step to finally achieve reliable quantitative data even for weak synaptic protein expression changes.

1. Dissolve synaptosomes or PSD-enriched preparations of each brain area of an animal in $20-50 \mu \mathrm{l}$ (dependent on total amount of material: for auditory cortex with $5-15 \mathrm{mg}$ tissue use $20 \mu \mathrm{l}$ ) of $8 \mathrm{M}$ urea and incubate on ice for $1 \mathrm{hr}$ in an ultrasonic bath.

1. For in-gel digest, dissolve synaptosomes directly in the SDS-sample buffer. Carefully calculate the loaded amount to avoid overload of the gel. Consider that in this case, the high abundant scaffold proteins will be lost during the gel electrophoresis and in-gel digest.

2. Dilute with $1 \%$ of a removable detergent to ensure a final concentration of $2 \mathrm{M}$ urea. Avoid any temperature higher than $30{ }^{\circ} \mathrm{C}$ to prevent protein carbamylation.

3. Perform SDS-PAGE with an aliquot (e.g. $10 \mu \mathrm{l})$ of the sample according to standard procedures ${ }^{13,14}$

4. Stain the gel with Coomassie Blue according to manufacturer's protocol. The procedure combines the fixing and staining step with methanol and acetic acid.

5. Determine the optical density of each sample for the whole lane with a calibrated gel scanner in transmission mode and calculate the relative protein amount.

6. Normalize the samples according to these calculations.

7. Split each sample into two different parts. Use one third for the in-gel digest and two thirds for the in-solution digest.

2. In-gel digest

1. Gel separation

1. Perform a second SDS-PAGE utilizing the concentration-adjusted samples. Stain and quantify the gels for a second time to check the normalization quality.

2. Cut out each lane of a sample within the gel in different areas (8/lane) but exclude the molecular weight range above $170 \mathrm{kDa}$. Transfer the gel pieces into separate tubes.

3. Cut the areas in smaller pieces (approx. $1 \times 1 \mathrm{~mm}$ ) with a sharp scalpel to facilitate in-gel digestion efficacy.

2. Digest ${ }^{15}$

1. Wash the gel pieces several times (depending on staining intensity) for 10 min with $50-150 \mu \mathrm{l}$ of a buffer consisting of $50 \%$ acetonitrile (ACN) and $50 \mathrm{mM}$ ammonium hydrogen carbonate $\left(\mathrm{NH}_{4} \mathrm{HCO}_{3}\right)$.

2. Remove supernatants. Cover the gel pieces with $\mathrm{ACN}$ and incubate at $20{ }^{\circ} \mathrm{C}$ until gel pieces become white and shrink.

3. Remove the $\mathrm{ACN}$ and rehydrate the gel pieces for $5 \mathrm{~min}$ with $50 \mu \mathrm{l}$ of $0.1 \mathrm{M} \mathrm{NH}_{4} \mathrm{HCO}_{3}$. Add the same volume of $\mathrm{ACN}$ and incubate for further $15 \mathrm{~min}$ at $37^{\circ} \mathrm{C}$ 
4. Remove and discard liquid completely. Dry the gel pieces in a vacuum centrifuge.

5. Rehydrate gel pieces in $50 \mu \mathrm{l}$ of $\mathrm{NH}_{4} \mathrm{HCO}_{3}$ containing $10 \mathrm{mM}$ dithiothreitol (DTT) and heat samples for $45 \mathrm{~min}$ at $56{ }^{\circ} \mathrm{C}$ to reduce cysteine residues.

6. Remove supernatants and add $50 \mu \mathrm{NH}_{4} \mathrm{HCO}_{3}$ containing $55 \mathrm{mM}$ iodoacetamide (IAA) for 30 min in the dark to carbamidomethylate reduced cysteines.

7. Remove and discard all liquid above the gel pieces and wash them twice with $50 \mu \mathrm{NH}_{4} \mathrm{HCO}_{3}$ and $\mathrm{ACN}(1: 1)$ for 10 min to remove any residual IAA. Dry samples in a vacuum centrifuge.

8. For limited digestion of proteins add $25 \mathrm{mM} \mathrm{NH}_{4} \mathrm{HCO}_{3}$ containing $12.5 \mathrm{ng} / \mu \mathrm{l}$ of trypsin. The required volume depends on size and amount of the gel pieces. Incubate for a few minutes and check if the buffer is absorbed. Add more buffer if necessary, gel pieces should be completely covered. Incubate at $37^{\circ} \mathrm{C}$ overnight (min. $12 \mathrm{hr}$ ).

3. Peptide extraction

1. Overlay gel pieces with $10-20 \mu \mathrm{l}$ of $25 \mathrm{mM} \mathrm{NH}_{4} \mathrm{HCO}_{3}$ and add the same volume of $\mathrm{ACN}$. Incubate for 10 min on ice using ultrasonic bath. Afterwards remove and collect supernatants which contain most of the generated peptides.

2. Add $100 \mu \mathrm{l}$ of extraction buffer containing $30 \% \mathrm{ACN} / 0.1 \%$ trifluoroacetic acid (TFA) to the gel pieces. Repeat incubation in an ultrasonic bath and carefully collect this supernatant.

3. Repeat the last extraction steps by increasing the ACN concentration to $50 \%$. After 10 min of ultrasonic bath spin down and collect supernatants.

4. Combine all three corresponding supernatants of the extraction steps and dry them in a vacuum centrifuge. Note that as a result of the gel separation the 8 areas per lane/sample are combined to one sample again in this step.

3. In-solution digest

1. Digest

1. Use the calculated amount (e.g. $100 \mu \mathrm{l}$ of a $150 \mu \mathrm{l}$ lysate, depends on the amount of material and the volume required for resuspension of a sample from a specific brain area) of normalized samples to obtain sufficient starting material for at least three technical replicates to perform label-free mass spectrometry.

2. Add $2 \mathrm{mM} \mathrm{DTT}$ in $25 \mathrm{mM} \mathrm{NH}_{4} \mathrm{HCO}_{3}$ and gently vortex the sample. Reduce the samples for $45 \mathrm{~min}$ at $20^{\circ} \mathrm{C}$.

3. Add $10 \mathrm{mM}$ IAA to carbamidomethylate the cysteine residues. Mix and incubate for $30 \mathrm{~min}$ in the dark at $20^{\circ} \mathrm{C}$.

4. Finally, add $1 \mu \mathrm{l}$ of a trypsin stock solution $\left(1 \mu \mathrm{g} / \mu \mathrm{l}\right.$ trypsin in $25 \mathrm{mM}$ acetic acid) and incubate at $20^{\circ} \mathrm{C}$ for $12 \mathrm{hr}$.

2. Solid-phase extraction (SPE)-Purification

1. To remove the acid cleavable detergent, adjust samples to a final concentration of $1 \%$ TFA and incubate for $1 \mathrm{hr}$ at $20^{\circ} \mathrm{C}$.

2. Centrifuge samples at $16,000 \times \mathrm{g}$ for $10 \mathrm{~min}$ and carefully collect supernatants.

3. Place the SPE column in a rack and equilibrate the matrix with $2 \mathrm{ml}$ methanol. Wash two times with $2 \mathrm{ml}$ of $0.1 \%$ TFA in water (buffer B).

4. Add $2 \mathrm{ml}$ of buffer $\mathrm{B}$ and load the sample. Wash another three times.

5. Elute the peptides by adding $200 \mu \mathrm{l} 70 \%$ ACN/0.1\% TFA. Repeat this step.

6. Pool both eluates and dry them down in a vacuum centrifuge.

4. Phospho-peptide-enrichment by $\mathrm{TiO}_{2}$ chromatography $^{16}$

1. Dissolve peptides produced by in-gel or in-solution digest in $150 \mu \mathrm{l}$ of $80 \% \mathrm{ACN} / 2.5 \% \mathrm{TFA}$ (buffer $\mathrm{C}$ ) and equilibrate $\sim 2 \mathrm{mg}$ of the $\mathrm{TiO}_{2}$ beads in $50 \mu$ l of buffer $C$.

2. Add beads to the sample and incubate in a rotating device for $1 \mathrm{hr}$ at $20^{\circ} \mathrm{C}$. Afterwards, spin beads down $(16,000 \times \mathrm{g}, 1 \mathrm{~min})$ and collect supernatants.

3. Wash the beads three times with $100 \mu$ l of buffer $C$ by gently mixing and spinning down after 5 min. Collect supernatants. Repeat this step three times with $100 \mu \mathrm{l}$ of $80 \%$ ACN/0.1\% TFA followed by three washes with $100 \mu \mathrm{l}$ of $0.1 \%$ TFA (without ACN), respectively.

4. Combine all ten supernatants, dry them in a vacuum centrifuge and handle them as the phospho-peptide-depleted fraction for further purification according step 3.5.

5. Elute the bound phospho-peptides with $20 \mu \mathrm{l}$ of $400 \mathrm{mM} \mathrm{NH}_{4} \mathrm{OH} / 30 \% \mathrm{ACN}$ from the beads. Repeat this step three times and collect all supernatants after spinning down the beads.

6. Combine the eluates of the in-gel digest and of the in-solution digest of a sample and handle them as the phospho-peptide-enriched fraction. Dry them in a vacuum centrifuge to a final volume of $4-8 \mu \mathrm{l}$.

5. Concentrating and desalting of phospho-peptide-depleted fractions by micro-SPE

1. Dissolve the dried peptides in $20 \mu$ of $0.1 \%$ TFA.

2. Equilibrate the fixed $\mathrm{C}_{18}$-matrix by drawing $20 \mu \mathrm{ACN}$ into the tip. Wash the matrix by drawing $0.1 \%$ TFA in water into the tip. Repeat the process three times.

3. Slowly load acidified sample into the tip (repeat this step three times).

4. Wash the $\mathrm{C}_{18}$-matrix three times with $20 \mu \mathrm{l} 0.1 \%$ TFA in water and discard the washing solution.

5. Elute peptides from the pipette tip by repeatedly ( 3 times) drawing $20 \mu \mathrm{l}$ of $70 \%$ ACN/0.1\% TFA and collect this elution solution in a separate tube.

6. Combine the eluates of a sample and dry them in a vacuum centrifuge.

\section{Proteome Analysis}

NOTE: Proteome analysis is performed on a hybrid dual-pressure linear ion trap/orbitrap mass spectrometer equipped with an ultra HPLC. The HPLC is composed of a cooled autosampler with a $20 \mu \mathrm{l}$ injection loop, a binary loading pump ( $\mu \mathrm{l}$ flow range), a binary nano flow separation pump, 
a column heater with two micro switching valves and a degasser. Samples are firstly subjected to a trapping column (e.g. $100 \mu \mathrm{m} \times 2 \mathrm{~cm})$ at a flow rate of $7 \mu \mathrm{l} / \mathrm{min}$ followed by separation on a column $(e . g .75 \mu \mathrm{m} \times 25 \mathrm{~cm})$ at $250 \mathrm{nl} / \mathrm{min}$. The separation column outlet is directly coupled to a coated Pico emitter tip positioned in a nano-spray interface at the mass spectrometer ionization source.

1. Nano-liquid chromatography and tandem mass spectrometry

1. Dissolve peptide samples in $12 \mu \mathrm{l} 2 \%$ ACN/0.1\% TFA for at least $30 \mathrm{~min}$. Spin down for $15 \mathrm{sec}$ and transfer $11 \mu \mathrm{l}$ supernatant to autosampler vials (conical, reduced diameter).

2. Set up an automated regime for sample application, chromatographic separation and tandem mass spectrometry at controlling software (e.g., Xcalibur) as follows.

1. Use the following for Temperature: Autosampler: $5^{\circ} \mathrm{C}$; Column oven: $45^{\circ} \mathrm{C}$.

2. Use the following for Injection: Volume: $10 \mu \mathrm{l}$; Flow rate: $7 \mu \mathrm{l} / \mathrm{min}(2 \% \mathrm{ACN}, 0.1 \% \mathrm{TFA})$; Time: 8 min; Valve setting: trap column waste; mass spec acquisition: off.

3. Use the following for Separation: Flow rate: $250 \mathrm{nl} / \mathrm{min}$ Valve setting: trap column-separation column; mass spec acquisition: on. 0 min - 100 min: $2 \%$ ACN, $0.1 \%$ formic acid $-40 \%$ ACN, $0.1 \%$ formic acid 100 min - 105 min: $40 \%$ ACN, $0.1 \%$ formic acid - $95 \%$ ACN, $0.1 \%$ formic acid

$105 \mathrm{~min}-109 \mathrm{~min}: 95 \% \mathrm{ACN}, 0.1 \%$ formic acid

109 min - 120 min: $2 \%$ ACN, $0.1 \%$ formic acid

4. Use the following for mass spectrometry settings: Full MS: FTMS; resolution 60,000; m/z range $400-2,000$; MS/MS: Linear Iontrap; minimum signal threshold 500; isolation width $2 \mathrm{Da}$; dynamic exclusion time setting $30 \mathrm{sec}$; singly-charged ions are excluded from selection; normalized collision energy is set to $35 \%$, and activation time to $10 \mathrm{msec}$.

NOTE: A full MS scan is followed by up to 15 LTQ MS/MS runs using collision-induced-dissociation (CID) of the most abundantly detected peptide ions.

3. Run three technical replicates for all samples.

2. Protein identification and label free quantification

1. Process mass spectrometric raw data towards protein identification and label-free quantification utilizing a commercial software suite (e.g., PEAKS Studio). In contrast to most other proteome software packages this particular software uses a de novo-sequencing algorithm prior to protein database alignments. However, this step can be easily substituted by other popular software packages.

2. Use essential settings listed in Table 2.

3. Phospho-proteomics

NOTE: Efficient and reliable phospho-peptide acquisition requires a few essential changes of the proteomic workflow setup.

1. After phospho-peptide enrichment, never dry samples completely. Always keep samples dissolved

NOTE: The phospho-ester bond of phosphorylated threonines or serines is very fragile. During collision-induced fragmentation within the ion trap this results in a neutral loss of phosphate. This prevents any further fragmentation of the peptide, which in turn is required for identification. Permitted wideband-activation in the mass spectrometry setup allows the fragmentation of phospho-peptides even after a neutral loss of the phosphate group. It performs a time saving "pseudo-MS ${ }^{3}$ ". Phospho-site determination in MS/MS data requires a particular verification and evaluation and can be performed by phosphoRS 3.0.

\section{Bioinformatics - Meta-Analysis}

NOTE: Before performing functional annotation and network analysis, the protein lists have to be preprocessed. First merge the lists of regulated proteins and phospho-peptides for each brain region separately. Then remove all duplicate UniProt-IDs for each fraction to prevent misinterpretation.

1. Singular enrichment analysis with GeneCodis ${ }^{17}$

1. Open the web-based tool of GeneCodis (http://genecodis.cnb.csic.es)

2. Select "Mus musculus" as organism and "GO Biological Process" as annotation.

3. Paste a list of UniProt-IDs of a certain fraction. Submit and wait until the analysis is performed. Click on "Singular Enrichment Analysis of GO Biological Process" and view results.

4. Repeat step 5.1.3 for the other three fractions.

5. To see any duplications and intersections between the result lists use a scripting language like Perl or Python to filter the data needed. Similar tools for a singular enrichment analysis are DAVID (https://david.ncifcrf.gov/) and Cytoscape (http://www.cytoscape.org/) with the Pluglns BiNGO (http://apps.cytoscape.org/apps/bingo) and ClueGO (http://apps.cytoscape.org/apps/cluego).

2. Generating a force based graph out of GeneCodis data with Gephi (https://gephi.org/)

NOTE: The data for the graphs has to be provided by the user, either in a graph format (.gexf, .graphml, .dot, .gv, .gml) or entered by hand.

1. Generating the graph nodes

1. By hand: Open Gephi and click on "Data Laboratory". Create nodes. Click on "Nodes" on the left to switch to the "Nodes" table. Click on "Add node". Enter the name of the Term. Click "OK"/Press Enter.

2. Alternative: Save GeneCodis result to PC. Open the .txt with a spreadsheet program. Delete all rows except from "Item_Details" (term names). Change header "Item_Details" to "Label". Save Spreadsheet as ".csv". Now in Gephi, click on "Import Spreadsheet". Choose spreadsheet from the file browser of Gephi. Click "Next". Click "Finish".

2. Connecting nodes via edges.

1. Click on "Edges" on the left to switch to the "Edges" table. For every node (Term): look up gene names in other Terms. If one or more genes are shared $->$ create edge. 
2. Click on "Add Edge". Select "Undirected". Select source and target node out of drop down lists. Click "OK"/Press Enter. If more than one gene is shared, enter abundance in "Weight" (table).

3. Force based graphical layout.

1. Open graph data file, set the graph type to "undirected" or use the data as entered by hand, click on "Overview" if not already selected.

2. Resize nodes depending on the abundance of interconnections. Click on Statistics, run either "Average Degree" (unweighted edges) or "Avg. Weighted Degree" (weighted edges) under "Network Overview". In "Appearance", click on "Nodes", then on the Size Button, next choose "Attributes" and set the Attributes parameter to "Avg. Weighted Degree" or "Average Degree". Click Apply.

3. Finally: Select "Force Atlas" in "Layout" and run; change "Repulsion strength" if nodes are colliding.

4. Export to picture.

1. Screenshot feature: Click on "Overview", change graph layout, edge thickness, label size and scaling with the menu at the bottom of "Graph" window. Click the camera left button, and save picture.

2. Export feature of "Preview": Click "Preview". Change Presets to "Default straight". Change Settings according the chosen preferences and click on "SVG/PDF/PNG" to export.

\section{Representative Results}

Figure 1 summarizes the complete workflow of quantitative synaptic proteome profiling of mouse brain regions after auditory discrimination learning. It starts with the animal training in a shuttle box. In the example shown in Figure 2, mice started to show significant FM tone discrimination in the $4^{\text {th }}$ training session, indicating efficient learning. Animals are sacrificed at selected time points for brain area dissection. The required enrichment of synapses can either be achieved by the preparation of synaptosomes or alternatively by the preparation of a PSDenriched fraction, both described in detail in Figure 3. The PSD-enrichment method has been developed for low tissue amounts, e.g. 1 - 2 hippocampal slices from rat brain ${ }^{12,18}$. It requires small tubes, PTFE pestles fitting to these tubes, and a laboratory drilling drive for powering the pestle.

Due to the particular protein composition of synaptosomes, it is strongly recommend to perform the sample preparation in two different but complementary ways. Scaffolds of the PSDs are often very high molecular weight proteins occurring in high stoichiometry. In-solution digest is the best way to extract them efficiently but may lead to an oversampling of the generated peptide mixture. The in-gel digest performed of the same sample in parallel can exclude those high molecular weight proteins and favor the analysis of proteins with medium and lower molecular weight. For a comprehensive analysis both types of proteolytic digests are recommended.

The different amounts of tissues of the brain areas investigated require an adjustment of the applied material for better comparison. Within the four investigated brain areas the auditory cortex is generally the limiting factor. The material of all other brain areas should carefully be adjusted to the amount of the auditory cortex after preparation of synaptosomes or PSD-enriched fractions (see 3.1.1.). Typical weights of freshly prepared brain areas from mice are as following: auditory cortex (AC): $50 \mathrm{mg}$; hippocampus (HIP): $90 \mathrm{mg}$; striatum (STR): $120 \mathrm{mg}$ and frontal cortex (FC): $100 \mathrm{mg}$.

The PSD-enrichment method described in section 2.3 allowed the identification of approximately 1500 different proteins and approximately 250 different phospho-peptides per brain region on the level of a single animal (Table 1). Proteomic analysis $24 \mathrm{~h}$ after the first training session revealed that $7.3 \%$ of the identified proteins and $5.8 \%$ of the phospho-peptides showed significant $(p<0.05)$ quantitative changes in their synaptic expression compared to naïve controls (Table 1). A conspicuous tendency for down regulation of synaptic scaffolds may point to a pronounced rearrangement of the synaptic architecture during early stages of FMTD learning. The vast majority of the regulated proteins were altered in a brain region-specific manner, whereas only $22 \%$ were found to be regulated in two or more brain areas. Six selected examples are shown in Figure 4.

Meta-analysis of the complex results by IPA provides evidence for the particular participation/manipulation of the following canonical pathways: "Clathrin-mediated Endocytosis Signaling", "Axonal Guidance Signaling", "Calcium Signaling", "RhoA Signaling", "Notch Signaling", "Remodeling of Epithelial Adherens Junctions", "Glutamate Receptor Signaling", "GABA Receptor Signaling", "Dopamine Receptor Signaling" and "Synaptic Long-Term Potentiation".

Single enrichment analysis revealed significant overrepresented biological processes in the frontal cortex concerning protein transport, cell adhesion, phosphorylation, endocytosis, vesicle-mediated transport, forebrain development and axonogenesis (Figure 5). In the auditory cortex biological processes including ion transport, translation, mRNA transport, protein transport and learning were noticeable. The analysis of the protein fraction of the hippocampus detects significantly enriched processes related to ion transport, cell cycle, translation, phosphorylation and nervous system development. In the striatum, overrepresented biological processes including mRNA transport, vesicle-mediated transport, axonogenesis, proteolysis, protein transport and endocytosis were found. 


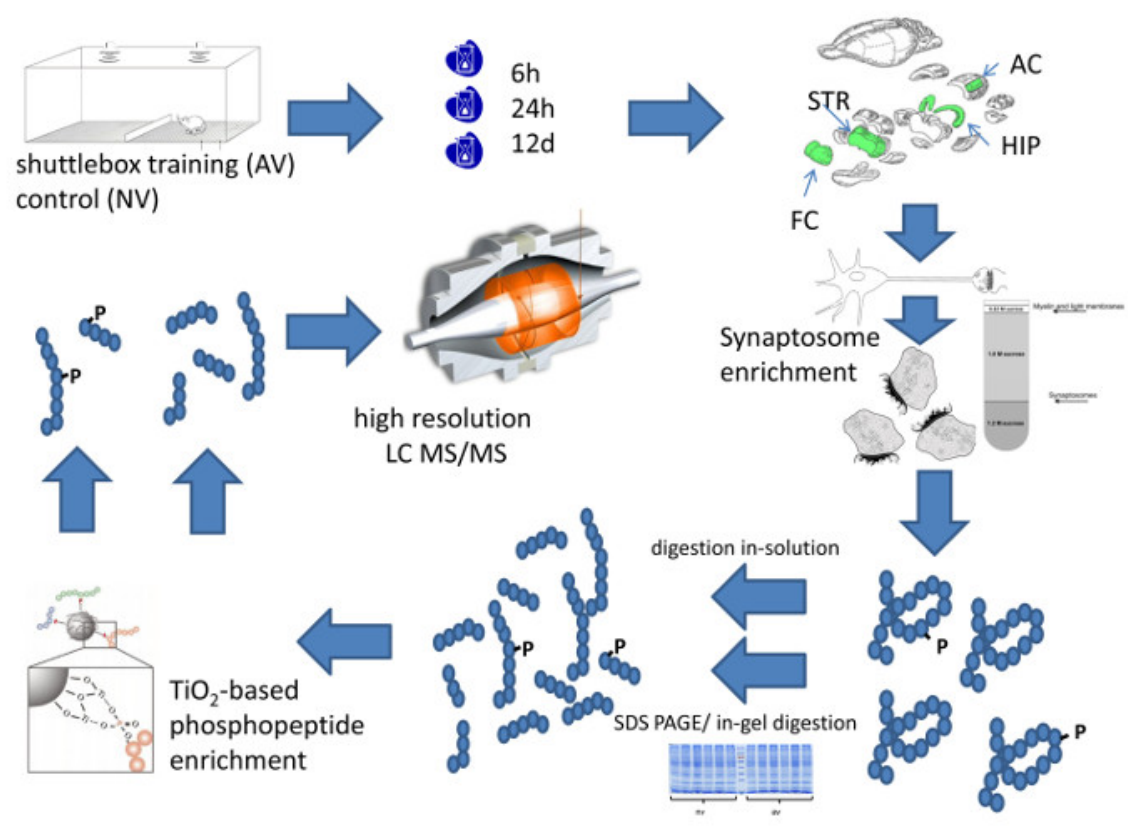

Figure 1: Systematic Workflow of the Methodological Approach. This figure schematically summarizes the workflow of high resolution quantitative profiling of brain area specific synaptic protein composition. Please click here to view a larger version of this figure.

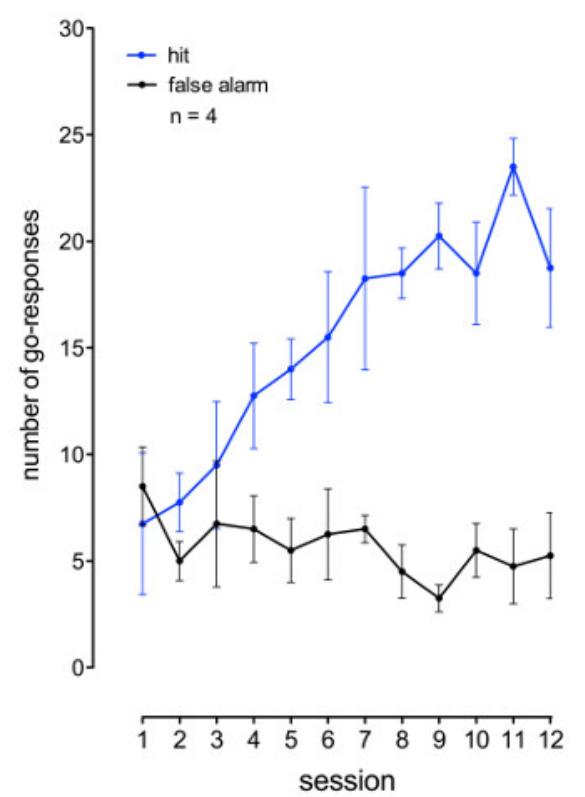

Figure 2: Example of the Performance of Mice in the FM Tone Discrimination Task. Animals show an increasing rate of hits (blue curve) and a decreasing rate of false alarms (black curve) in the course of training sessions. Significant discrimination occurs from the fourth session. Error bars are provided as SEM. Please click here to view a larger version of this figure. 


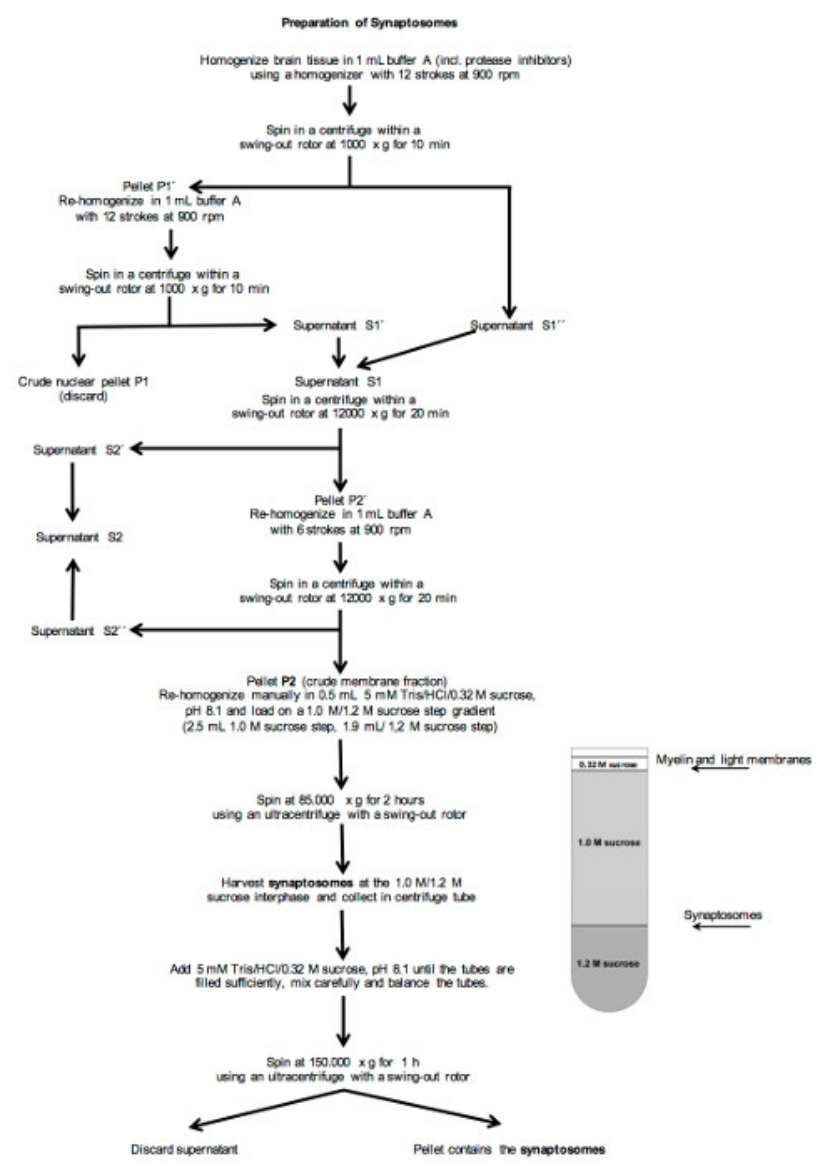

Figure 3: Preparation of the Synaptosome and the PSD-enriched Fraction. A: Synaptosome preparation. B: PSD-enriched fraction preparation. Both figures explain the detailed workflow of preparation of synaptosomes or alternatively PSD-enriched fractions from brain tissues. Please click here to view a larger version of this figure. 


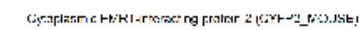
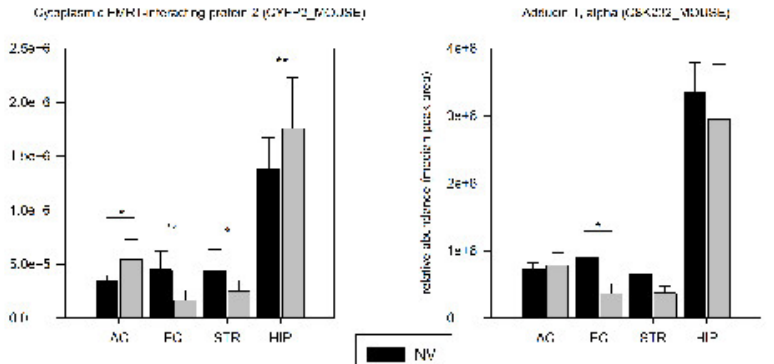

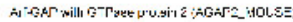

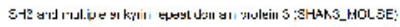
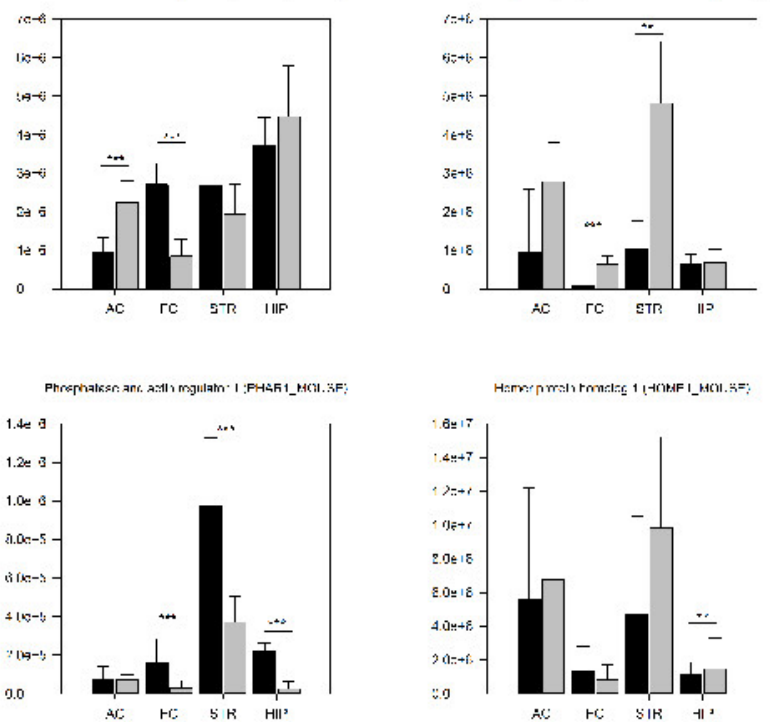

Figure 4: Selected Quantitative Proteomic Results. The relative synaptic abundances of selected proteins are compared between mice trained on the FMTD task (AV, n= 6) and naïve control mice $(N V, n=6) 24 \mathrm{hr}$ after the first training session. The abundance values were calculated as median of the peak areas of the three most intense peptides of a protein. Proteins with significant abundance changes (AV/NV; $t-$ test) are marked within the plots: ${ }^{*} p<0.05,{ }^{* *} p<0.01,{ }^{* * *} p<0.005$. Error bars are provided as SD. Please click here to view a larger version of this figure. 


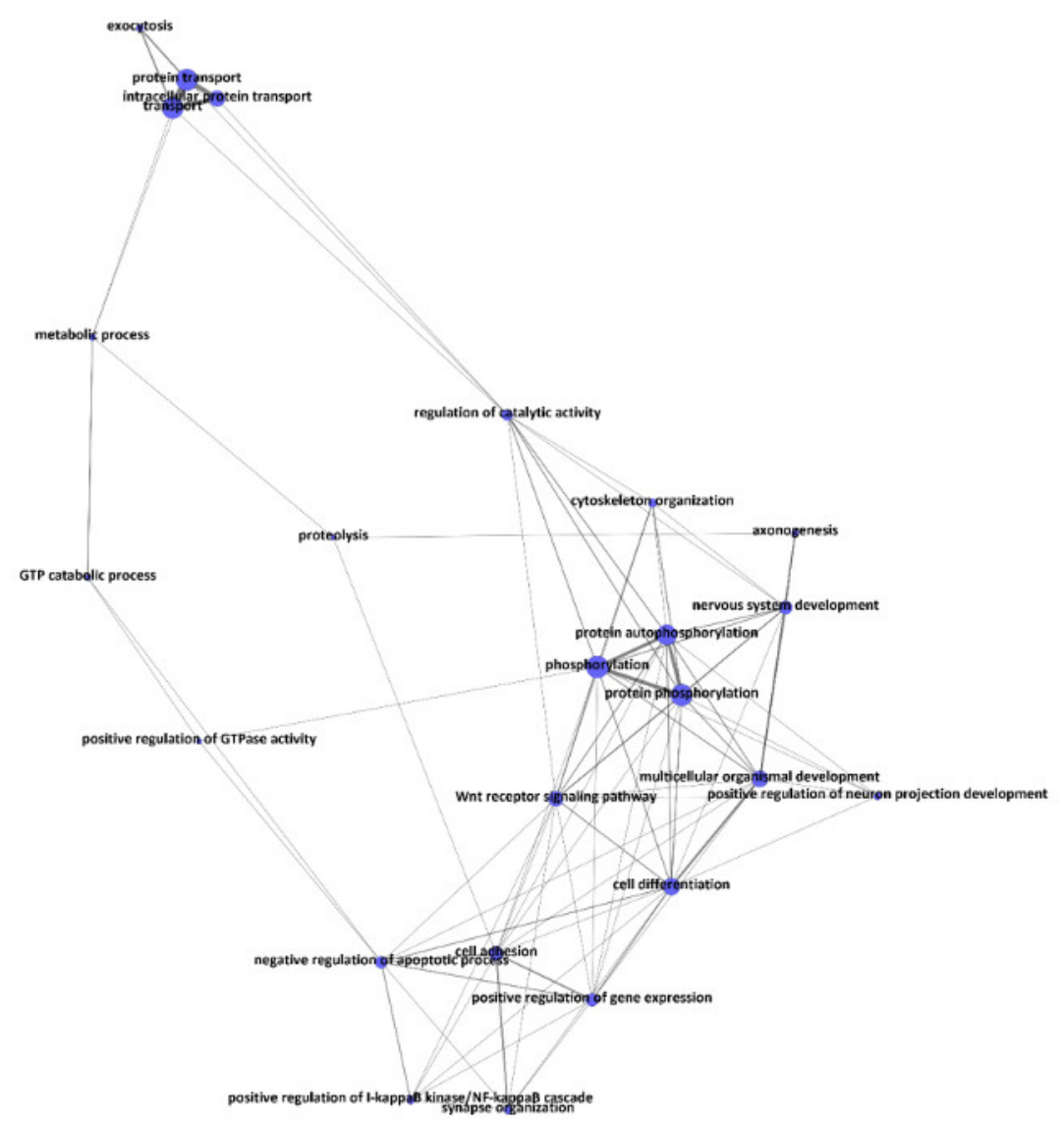

Figure 5: Visualization of Biological Pathways for Frontal Cortex by GeneCodis/Gephi. Only significant terms of the Gene Ontology (GO) database (http://geneontology.org) related to "Biological process" with a minimum protein number of three are shown here. Nodes represent GO terms, the size of the node, the line width and number of connections of a certain node depict the number of proteins, which share this GO term with other nodes. Due to the "Force Atlas" method of Gephi, related nodes are clustering closely together. Please click here to view a larger version of this figure.

\begin{tabular}{|c|c|c|c|c|c|}
\hline Brain region & $A C$ & FC & HIP & STR & $\#$ \\
\hline identified proteins & 1435 & 1758 & 1572 & 1507 & 6272 \\
\hline $\begin{array}{l}\text { regulated proteins } \\
(\mathrm{p}<0.05)\end{array}$ & 59 & 130 & 162 & 108 & 459 \\
\hline \# AV/NV & 8 & 4 & 76 & 35 & 123 \\
\hline \# AV/NV & 51 & 126 & 86 & 73 & 336 \\
\hline $\begin{array}{l}\text { identified } \\
\text { phosphomotifs }\end{array}$ & 197 & 361 & 273 & 278 & 1109 \\
\hline $\begin{array}{l}\text { regulated } \\
\text { phosphomotifs } \\
(\mathrm{p}<0.05)\end{array}$ & 8 & 22 & 21 & 14 & 65 \\
\hline \# AV/NV & 4 & 17 & 5 & 9 & 35 \\
\hline \# AV/NV & 4 & 5 & 16 & 5 & 30 \\
\hline
\end{tabular}

Table 1: Summary of a Proteomic Result. This table summarizes a representative proteomic experiment of trained mice (AV, $n=6) 24 \mathrm{hr}$ after the first training session compared to their naïve controls $(N V, n=6)$. The sum of 459 regulated proteins includes overlapping regulations. 283 different regulations were determined as brain specific. In detail, 57 proteins are regulated in two brain regions, 18 protein regulations were detected in three brain regions and only 2 proteins are regulated in all four investigated brain areas. 


\begin{tabular}{|c|c|}
\hline Error tolerances & \\
\hline precursor mass (fourier transformation mass spectrometry) & $10 \mathrm{ppm}$ \\
\hline fragment ion mass (linear ion trap) & $0.6 \mathrm{Da}$ \\
\hline Maximum missed cleavages per peptide & 3 \\
\hline \multicolumn{2}{|l|}{ Fixed modifications } \\
\hline for in-gel-digested samples & Carbamidomethylation of Cysteine \\
\hline for in-solution-digested samples & Methylthiolation of Cysteine \\
\hline \multirow[t]{2}{*}{ Variable modifications } & Oxidation of Methionine \\
\hline & Deamidations of Asparagin and/or Glutamine \\
\hline Database & Uniprot/Sprot \\
\hline Taxonomy & mouse \\
\hline \multicolumn{2}{|l|}{ Statistical identification-acceptance settings } \\
\hline de novo average local confidence (ALC) & $>50 \%$ \\
\hline Peptide-false discovery rate (FDR, based on est. decoy-fusion) & $<1 \%$ \\
\hline Protein significance (-10logP, based on modified T-test) & $>20$ \\
\hline unique peptides / protein & $\geq 1$ \\
\hline \multirow{2}{*}{\multicolumn{2}{|c|}{ Quantification settings: }} \\
\hline & \\
\hline Peptide significance (-10logP) & $>30$ \\
\hline Peptide identification in & $\geq 50 \%$ of samples \\
\hline Peptide signal quality & $>1$ \\
\hline Peptide average area & $>1 \mathrm{E} 5$ \\
\hline Peptide retention time tolerance & $<5 \min$ \\
\hline Normalization & by total ion current (TIC) \\
\hline
\end{tabular}

Table 2: Settings for Protein Identification (step 4.2.2).

\section{Discussion}

The study presents a methodological workflow optimized for an accurate quantitative profiling of synaptic protein expression changes during learning and memory consolidation in different brain areas of mice. The setup provides the opportunity to study the protein expression on the level of a single animal despite of the required application of at least three technical replicates per sample for mass spectrometric analysis.

The methodology takes into account the particular protein composition of the pre- and postsynapse consisting of high molecular weight scaffold proteins but also of important mediator proteins bearing medium or lower molecular weights. The in-solution digests of synaptosomal preparations result in an efficient generation and, hence, an over-representation of scaffold-derived peptides. This, in turn, may suppress the analysis of smaller or lower abundant proteins. The suggested preparation of SDS-PAGE fractions from an aliquot of each sample combined with an in-gel digestion procedure in parallel facilitates the analysis of medium and low abundance proteins and represents a highly recommended complementary method. After separate mass spectrometric application of all fractions derived from a sample (e.g. in-solution digest, ingel digest, combined phospho-enriched fractions) the corresponding MS/MS data sets can be combined and further calculated for protein identification and quantification by PEAKS software or alternative popular software packages.

Alternatively, the individual application of in-gel-digestion-derived fractions of a sample (separately processed gel-areas of a sample lane) and fractions generated of the in-solution digested sample (e.g. by ion exchange chromatography) to mass spectrometry can increase the analytical depth. However, this extended workflow dramatically increases the required time for LS-MS/MS data acquisition. For generation of a detailed molecular sequence of synaptic protein rearrangements during learning and memory formation a specified time course of the proteomic profiling is required. This time course may start immediately after or even during the first training session and covers a close-meshed time frame until the animals' performance reached the asymptotic level of the learning curve after approx. 8 - 10 days of training (see Figure 2 for details).

The analysis of phosphorylation changes of synaptic proteins requires a particular focus on the selected time frames during FMTD learning. On the one hand signaling cascades initiating synaptic protein rearrangements known to be triggered by protein phosphorylations and dephosphorylations are expected at very early stages of animal training. On the other hand, there are long lasting modifications of multiple phosphorylated synaptic proteins known which regulate the connectivity and assembly within the synaptic architecture ${ }^{19,20}$. Those posttranslational modifications are expected even at later time points of memory consolidation. 
The complex datasets generated by this proteomic workflow require bioinformatic processing to identify participating molecular pathways and key molecules. Meta-analysis shows significant overrepresented pathways, which play a role in learning and memory processes.

\section{Disclosures}

The authors have nothing to disclose.

\section{Acknowledgements}

We wish to thank Yvonne Ducho and Kathrin Pohlmann for excellent technical assistance. This work was supported by the Deutsche Forschungsgemeinschaft (SFB 779) and by the State Saxony-Anhalt / European Regional Development Fund (ERDF) via the Center for Behavioral Brain Sciences (CBBS).

\section{References}

1. Lamprecht, R., \& LeDoux, J. Structural plasticity and memory. Nat Rev Neurosci. 5 (1), 45-54, (2004)

2. Bingol, B., \& Schuman, E. M. Synaptic protein degradation by the ubiquitin proteasome system. Curr Opin Neurobiol. 15 (5), $536-541$ (2005).

3. Richter, J. D., \& Klann, E. Making synaptic plasticity and memory last: mechanisms of translational regulation. Genes Dev. 23 (1), 1-11 (2009).

4. Rosenberg, T. et al. The roles of protein expression in synaptic plasticity and memory consolidation. Front Mol Neurosci. 7, 86 (2014).

5. Scheich, H. et al. Behavioral semantics of learning and crossmodal processing in auditory cortex: the semantic processor concept. Hear Res. 271 (1-2), 3-15 (2011).

6. Kähne, T. et. al. Synaptic proteome changes in mouse brain regions upon auditory discrimination learning. Proteomics. 12 (15-16), 2433-2444 (2012).

7. Reichenbach, N. et al. Differential effects of dopamine signalling on long-term memory formation and consolidation in rodent brain. Proteome Sci. 13, 13 (2015).

8. Kähne, T. et. al. Proteome rearrangements after auditory learning: high-resolution profiling of synapse-enriched protein fractions from mouse brain. J Neurochem. (2016).

9. $\mathrm{Li}, \mathrm{K}$. et al. Organelle proteomics of rat synaptic proteins: correlation-profiling by isotope-coded affinity tagging in conjunction with liquid chromatography-tandem mass spectrometry to reveal post-synaptic density specific proteins. J Proteome Res. 4 (3), 725-733 (2005)

10. Carlin, R. K., Grab, D. J., Cohen, R. S., \& Siekevitz, P. Isolation and characterization of postsynaptic densities from various brain regions: enrichment of different types of postsynaptic densities. J Cell Biol. 86 (3), 831-845 (1980).

11. Smalla, K. H., Klemmer, P., \& Wyneken, U. in The Cytoskeleton - Imaging, Isolation, and Interaction. (ed R. Dermietzel) 265-282 Humana Press, (2012).

12. Smalla, K. H. et al. The synaptic glycoprotein neuroplastin is involved in long-term potentiation at hippocampal CA1 synapses. Proc Natl Acad Sci U S A. 97 (8), 4327-4332 (2000).

13. Shapiro, A. L., Vinuela, E., \& Maizel, J. V., Jr. Molecular weight estimation of polypeptide chains by electrophoresis in SDS-polyacrylamide gels. Biochem Biophys Res Commun. 28 (5), 815-820 (1967).

14. Laemmli, U. K. Cleavage of structural proteins during the assembly of the head of bacteriophage T4. Nature. 227 (5259), $680-685$ (1970).

15. Shevchenko, A., Wilm, M., Vorm, O., \& Mann, M. Mass spectrometric sequencing of proteins silver-stained polyacrylamide gels. Anal Chem. 68 (5), 850-858 (1996).

16. Thingholm, T. E., Larsen, M. R., Ingrell, C. R., Kassem, M., \& Jensen, O. N. TiO(2)-based phosphoproteomic analysis of the plasma membrane and the effects of phosphatase inhibitor treatment. J Proteome Res. 7 (8), 3304-3313 (2008).

17. Carmona-Saez, P., Chagoyen, M., Tirado, F., Carazo, J. M., \& Pascual-Montano, A. GENECODIS: a web-based tool for finding significant concurrent annotations in gene lists. Genome Biol. 8 (1), R3 (2007).

18. Bonn, S., Seeburg, P. H., \& Schwarz, M. K. Combinatorial expression of alpha- and gamma-protocadherins alters their presenilin-dependent processing. Mol Cell Biol. 27 (11), 4121-4132 (2007).

19. Tweedie-Cullen, R. Y., Reck, J. M., \& Mansuy, I. M. Comprehensive mapping of post-translational modifications on synaptic, nuclear, and histone proteins in the adult mouse brain. J Proteome Res. 8 (11), 4966-4982 (2009).

20. Li, L. et al. The differential hippocampal phosphoproteome of Apodemus sylvaticus paralleling spatial memory retrieval in the Barnes maze. Behav Brain Res. 264, 126-134 (2014). 